\title{
UNIVERSITYOF
}

FORWARD

THINKING

WESTMINSTER用

WestminsterResearch

http://www.westminster.ac.uk/westminsterresearch

\section{A Fuzzy-Wavelet Neural Network Model for the Detection of Meat Spoilage using an Electronic Nose}

Kodogiannis, V. and Alshejari, A.

This is a copy of the author's accepted version of a paper subsequently published in the proceedings of the IEEE International Conference on Fuzzy Systems (FUZZ-IEEE), Vancouver, BC, Canada 24 to 29 Jul 2016, IEEE.

The final definitive is available online at:

\section{https://dx.doi.org/10.1109/FUZZ-IEEE.2016.7737757}

(C) 2016 IEEE . Personal use of this material is permitted. Permission from IEEE must be obtained for all other uses, in any current or future media, including reprinting/republishing this material for advertising or promotional purposes, creating new collective works, for resale or redistribution to servers or lists, or reuse of any copyrighted component of this work in other works.

The WestminsterResearch online digital archive at the University of Westminster aims to make the research output of the University available to a wider audience. Copyright and Moral Rights remain with the authors and/or copyright owners.

Whilst further distribution of specific materials from within this archive is forbidden, you may freely distribute the URL of WestminsterResearch: ((http://westminsterresearch.wmin.ac.uk/)).

In case of abuse or copyright appearing without permission e-mail repository@westminster.ac.uk 


\title{
A Fuzzy-Wavelet Neural Network Model for the Detection of Meat Spoilage using an Electronic Nose
}

\author{
Vassilis S. Kodogiannis, Abeer Alshejari \\ Faculty of Science and Technology \\ University of Westminster \\ London, United Kingdom \\ V.Kodogiannis@westminster.ac.uk
}

\begin{abstract}
Food product safety is one of the most promising areas for the application of electronic noses. The performance of a portable electronic nose has been evaluated in monitoring the spoilage of beef fillet stored aerobically at different storage temperatures $\left(0,4,8,12,16\right.$ and $\left.20^{\circ} \mathrm{C}\right)$. This paper proposes a fuzzy-wavelet neural network model which incorporates a clustering pre-processing stage for the definition of fuzzy rules. The dual purpose of the proposed modeling approach is not only to classify beef samples in the respective quality class f.e. fresh, semi-fresh and spoiled), but also to predict their associated microbiological population directly from volatile compounds fingerprints. Comparison results indicated that the proposed modeling scheme could be considered as a valuable detection methodology in food microbiology.
\end{abstract}

Keywords-fuzzy systems; wavelet; neural networks; meat spoilage; clustering

\section{INTRODUCTION}

The resolution of the Uruguay Round of the General Agreement on Tariffs and Trade (GATT) in 1995, recognized public health risk as the only basis for restrictions of international trade in food. Beef is one of the commercially viable and widely consumed muscle foods throughout the world. Although it is a good food source for proteins and other essential nutrients, it is also an ideal substrate for the growth of both spoilage and pathogenic microorganisms. The current practice to assure the safety of meat still relies on regulatory inspection and sampling regimes. This approach, however, seems inadequate because it cannot sufficiently guarantee consumer protection, since $100 \%$ inspection and sampling is technically, financially and logistically impossible. Meat industry however needs rapid and non-destructive sensing methods for quantification of these indicators in order to determine suitable processing procedures for their raw material and to predict the remaining shelf life of their products [1].

Rapid and non-invasive methods based on analytical instrumental techniques, such as Fourier transform infrared spectroscopy (FTIR) [2] and Raman spectroscopy [3] have been considered for their potential in meat quality assessment. The "mechanism" of these approaches is based on the assumption that the metabolic activity of micro- organisms on meat results in biochemical changes, with the simultaneous formation of metabolic by-products, which may contribute to the spoilage phenomenon. The quantification of these metabolic activities corresponds to a unique "signature", providing thus information about the type and rate of spoilage [4].

The electronic nose (enose) is a system initially created to imitate the function of human nose. There are three primary components in an electronic nose: an array of chemical gas sensors with broad and partly overlapping selectivity that measure volatile compounds, a signal preparation system, and a pattern recognition system [5]. Although this technique does not allow the identification of compounds and has a high detection limit in comparison with GC-MS, it has been successfully used in processing monitoring, shelf-life investigation, freshness evaluation and authenticity assessment in a wide range of food products, including meat products.

One of the earliest research studies in the application of enose to meat quality analysis was conducted by Balasubramanian, where the changes in the headspace of vacuum packaged beef strip sides vaccinated with Salmonella typhimurium were evaluated using a metal oxide based enose [6]. The prediction of total viable counts (TVC) in chilled pork using an enose using support vector machine (SVM) has been investigated [7]. Enose has been also used for the development of a prediction model to detect the content of pork in minced mutton. The adulteration issue is an important problem for food safety [8].

The main objective of this paper is to associate acquired volatile fingerprints (snapshots) of odor profile with beef spoilage during aerobic storage at various temperatures $(0,4$, $8,12,16$ and $20^{\circ} \mathrm{C}$ ) through the development of an advanced intelligent-based decision support system. Datasets related to enose data as well as the associated microbiological analysis (i.e. TVC) from beef fillets, were provided by the Agricultural University of Athens, Greece. The achievement of this objective, however, involves the implementation of a number of sub-tasks, related to data analysis. Due to the multi-variable nature of enose data, a dimensionality reduction algorithm was applied on the data used for training purposes. The robust PCA (RPCA) scheme 
has been utilized to obtain principal components that are not influenced much by outliers [9]. In this study, a MIMO Clustering Fuzzy Wavelet Neural Network system (CFWNN) has been developed to predict the microbial load (as TVC) on meat surface, based on the biochemical profile provided by the enose dataset. In addition, the same model can classify beef samples to one of three quality classes (i.e. fresh, semi-fresh, and spoiled). The proposed CFWNN model differs from conventional fuzzy rule-table approaches that utilize the "look-up table" concept. In the case of CFWNN, a clustering algorithm is applied for the sample data in order to organize feature vectors into clusters such that points within a cluster are closer to each other than vectors belonging to different clusters. Then a fuzzy rule base is created using results obtained from this algorithm. Prediction results are compared with those produced by models based on ANFIS, and multilayer neural networks (MLP). Such comparison is considered essential, as we have to emphasize the need of induction to the area of food microbiology, advanced learning-based modeling schemes, which may have a significant potential for the rapid and accurate evaluation of meat spoilage.

\section{ENOSE SAMPLING AND ANALYSIS}

The experimental case study was performed at the Agricultural University of Athens, Greece. A detailed description of the experimental methodology, as well as the related microbiological analysis of the meat samples, is described in [10]. Briefly, the samples were prepared by cutting fresh pieces of beef into small portions and then packed aerobically in trays that were wrapped with airpermeable plastic film. Samples were stored under controlled isothermal conditions at $0,4,8,12,16$ and $20^{\circ} \mathrm{C}$ in high precision incubators for up to $434 \mathrm{~h}$, depending on storage temperature, until spoilage was apparent. At the beginning and during storage, after appropriate time intervals, duplicate meat samples were taken for microbiological, sensory and chemical analysis via enose.

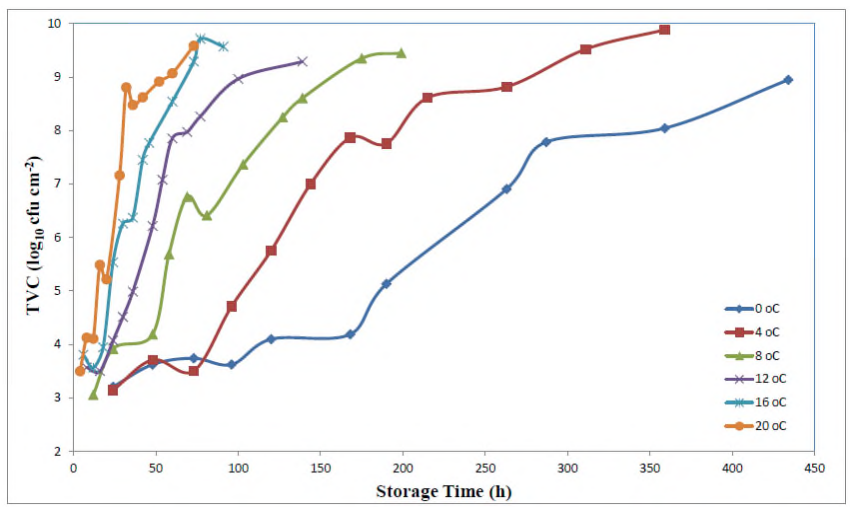

Fig. 1. Population dynamics of TVC at various temperatures

In parallel, microbiological analysis was performed, and resulting growth data from agar plate counts were $\log _{0}$ transformed and fitted to the primary model of Baranyi in order to verify the kinetic parameters of microbial growth (maximum specific growth rate and lag phase duration) [10]. The growth curves of total viable counts (TVC) for beef fillet storage at different temperatures under aerobic conditions are illustrated in Fig. 1. Additionally, sensory evaluation of meat samples was performed during storage, based on the perception of color and smell before and after cooking [10]. Each sensory attribute was assigned to a three-point scale corresponding to: $1=$ fresh (acceptable meat quality and the absence of off-flavors); $2=$ semi-fresh (presence of slight offflavors but not spoiled); and $3=$ spoiled (clearly off-flavor development). In total, 210 meat samples were evaluated by a sensory panel and classified into the selected three groups as fresh $(n=48)$, semi-fresh $(n=72)$, and spoiled $(n=90)$.

Libra enose is a compact analytical device used to classify and identify complex odors produced by Technobiochip [11]. The instrument is composed by an array of sensors and a data analysis system. Sensors work like biological receptors and data analysis system allows to transpose information that sensors extract from an odor in an "olfactory image" analogous with our "sensation" of a smell. Libra enose uses a set of eight $20 \mathrm{MHz}$ piezoelectric transducers placed in a measuring chamber. Fig 2 illustrates its details. The surface of each transducer is covered by a different poly-pyrrole derivatives layer which forms nonspecific bindings with the compounds of gas mixtures. This nonspecific binding makes sensors non-selective and prohibits them to be poisoned during measurements.

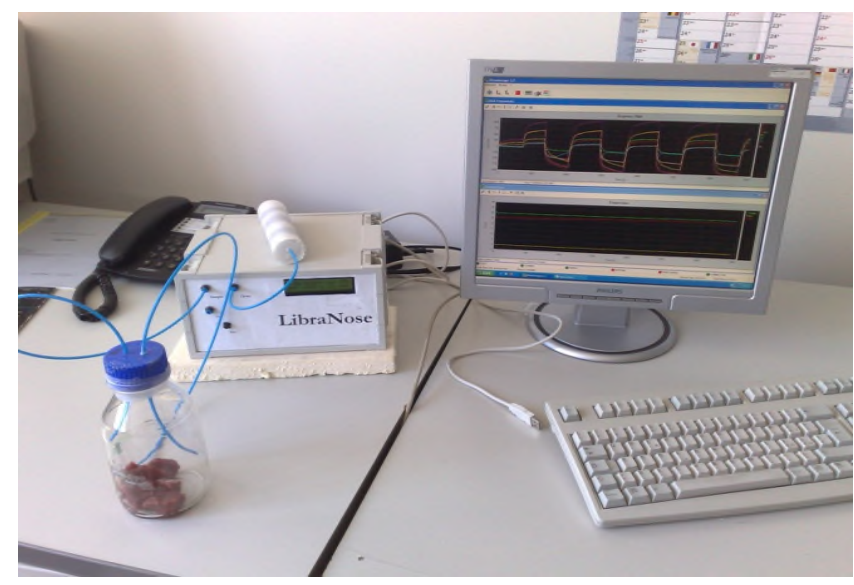

Fig. 2. Libra Electronic Nose

The device can be quickly reused after a short cycle of cleaning using clean filtered air obtained via a carbon active filter. The measuring chamber is held at a constant temperature during the measurements by a thermostatic electronic system. A flow system formed by a micro-electric valve and a micro-pump conveys the gas sample to the measuring chamber in a controlled, by the connected computer, way. For each measurement, a beef fillet sample of $5 \mathrm{~g}$ was introduced inside a $100 \mathrm{ml}$ volume glass jar and left at room temperature $\left(20^{\circ} \mathrm{C} \pm 2^{\circ} \mathrm{C}\right)$ for $15 \mathrm{~min}$ to enhance desorption of volatile compounds from the meat into the headspace. Pre-processing of the data obtained from enose sensors is required to obtain the "olfactory image" of the sample. This process involves extracting certain significant characteristics from the sensor response curves in order to produce a set of data that can be processed by the recognition system of the enose. Different features can be extracted and used depending on the characteristics of the enose used such as the type of sensors adopted, and the stability of the responses of the latter to the reference gas, to variations in 
humidity and temperature levels. The responses of all sensor signals classes for meat samples stored at $4{ }^{\circ} \mathrm{C}$ are shown in Fig. 3.

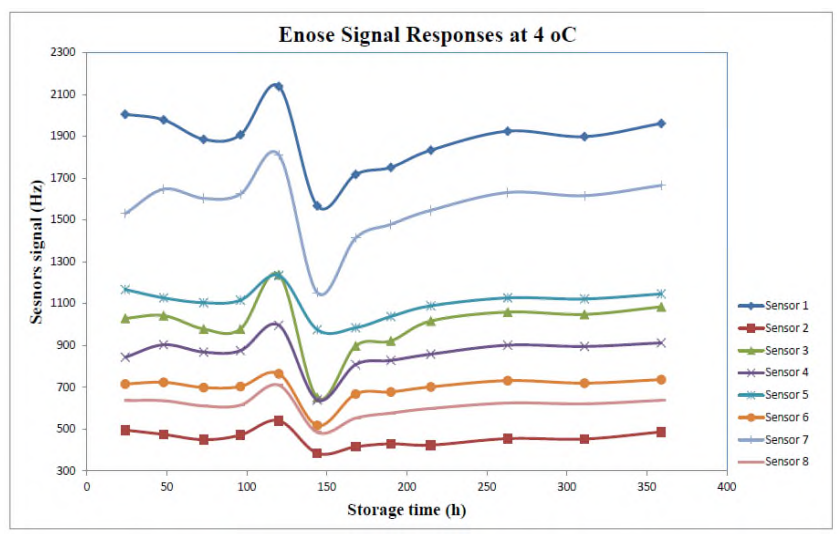

Fig. 3. Enose responses during storage of beef fillets at $4^{\circ} \mathrm{C}$

Considering that each measurement can be represented as a point in an 8-dimensional space, a dimensionality reduction algorithm has been applied on those enose data used for training purposes. The robust PCA (RPCA) scheme has been utilized to obtain principal components that are not influenced much by outliers. The RPCA procedure is implemented in three main steps. First, the data were preprocessed such that the transformed data are lying in a subspace whose dimension is at $\operatorname{mos} t n-1$. A preliminary covariance matrix was then constructed and used for selecting the number of components $k$ that will be retained in the sequel, yielding a $k$-dimensional subspace that fits the data well. Then the data points were projected on this subspace where their location and scatter matrix are robustly estimated, from which its $k$ nonzero eigenvalues $l_{1}, \ldots, l_{k}$ are computed. The corresponding eigenvectors are the $k$ robust principal components [9]. RPCA scheme was implemented in MATLAB, with the aid of PLS_Toolbox (ver. 8.0 Eigenvector.com).

TABLE I. ROBUST PCA SCHEME

\begin{tabular}{|c|c|c|c|}
\hline \multirow{2}{*}{ PCs } & \multicolumn{3}{|c|}{ Robust PCA } \\
\cline { 2 - 4 } & Eigenvalue & Prop. \% & Cum. prop. \% \\
\hline 1 & $7.17 \mathrm{e}+004$ & 71.45 & 71.45 \\
\hline 2 & $1.11 \mathrm{e}+004$ & 21.88 & 93.34 \\
\hline 3 & $2.40 \mathrm{e}+003$ & 4.11 & 97.45 \\
\hline 4 & $9.47 \mathrm{e}+002$ & 1.55 & 99.01 \\
\hline 5 & $2.70 \mathrm{e}+002$ & 0.50 & 99.50 \\
\hline
\end{tabular}

For this particular experimental case study, the first four principal components (PC) were associated with the $99 \%$ of the total variance, as shown in Table I. These specific PCs were extracted and utilized as inputs to the various simulation models developed for this specific case study.

\section{CFWNN ARCHITECTURE}

Generally, the FWNN is a combined structure based on fuzzy rules that includes wavelet functions in their consequent parts, in the form of a wavelet neural network. In these FWNN schemes, such combination is achieved through a Takagi-Sugeno-Kang (TSK) structure, which allows us to develop a system that has fast training speed, and describe nonlinear objects that are characterized with uncertainty. The domain interval of each input is separated into fuzzy regions and each region is associated with a membership function (MF) in the IF part of the fuzzy rules. The rules are then defined either by experts or are learnt adaptively similarly to ANFIS scheme [12].

In this research study, a novel Multi-Input Multi-Output (MIMO) Clustering Fuzzy Wavelet Neural Network (CFWNN) is proposed for the detection of meat spoilage. In the general case of FWNNs, the number of fuzzy rules is important as it affects the accuracy and the efficiency of the developed prediction system. A second problem is related to the initialization stage. In fact the initial parameters of the network greatly affect the accuracy result, the time required for learning and even the convergence and stability of the training process.

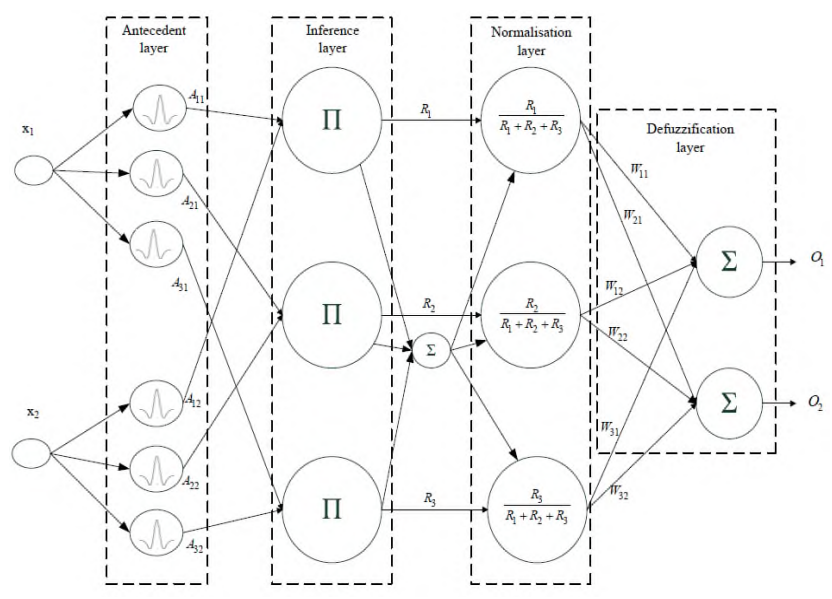

Fig. 4. CFWNN architecture

A clustering algorithm is applied initially, as a preprocessing step, to the training dataset in order to organize feature vectors into clusters, such that points within a cluster are closer to each other than vectors belonging to different clusters. The fuzzy rule base is derived using results obtained from a clustering algorithm. In the proposed scheme, the number of memberships for each input variable is directly associated to the number of rules, hence, the "curse of dimensionality" problem is significantly reduced. Fig. 4 illustrates the architecture of the MIMO CFWNN architecture.

Gaussian membership functions (MF) are commonly used in Neuro-Fuzzy (NF) systems. A deficiency of Gaussian-based NF networks is their limited ability to localize in the frequency domain. By comparison, the proposed CFWNN, where wavelet functions are utilized, has the ability to localize in both the time and frequency domains. In this paper, the following generalized Mexican Hat wavelet function with translation $(\mu)$ and dilation $(\sigma)$ parameters has been considered as MF:

$$
\psi\left(\frac{x-\mu}{\sigma}\right)=\left(1-\left(\frac{x-\mu}{\sigma}\right)^{2}\right) \exp \left(-0.5\left(\frac{x-\mu}{\sigma}\right)^{2}\right)
$$


Translation parameter determines the center position of the wavelet, while dilation parameter controls the spread of the wavelet. As MF values cannot be negative and larger than unity, the Mexican Hat MF has been normalized as follows:

$A_{i j}=\frac{\left(1-\left(\frac{x_{i}-\mu_{i j}}{\sigma_{i j}}\right)^{2}\right) \exp \left(-0.5\left(\frac{x_{i}-\mu_{i j}}{\sigma_{i j}}\right)^{2}\right)+\varepsilon}{1+\varepsilon}$

where constant $\varepsilon=0.446$. The structure of the CFWNN is explained below layer by layer:

- Layer 1: This layer is simply the input layer. Nodes in this layer pass on the input signals $x_{1}, x_{2}, \ldots, x_{n}$ to $\mathrm{L}_{2}$.

- Layer 2: This layer is the fuzzification layer, and its nodes represent the fuzzy sets used in the antecedent parts of the fuzzy rules. A fuzzification node receives an input and determines the degree to which this input belongs to in the node's fuzzy set. The outputs of this layer are the values of wavelet MFs for the input values. The normalised Mexican Hat MF $A_{i j}$ presented at Eq. 2 have been utilized for the proposed CFWNN, where, index $j$ is associated with the input variable, while index $i$ is linked with MF's $j^{\text {th }}$ input. The initial translation variables $\mu_{i j}$ at Eq. 3 are equal to the values of the components of the vectors $\boldsymbol{v}_{\boldsymbol{i}}$, which come from the second stage of the clustering pre-processing step. The dilation values $\sigma_{i j}$ are initialised according to

$\sigma_{i j}=\left(\sum_{k=1}^{n} u_{i k}\left(x_{k j}-v_{i j}\right)^{2} / \sum_{k=1}^{n} u_{i k}\right)^{1 / 2}$

These values are calculated based on the matrix $\mathbf{U}$, where its elements correspond to the fuzzy memberships of $\boldsymbol{x}_{\boldsymbol{k}}$ in the $i^{\text {th }}$ cluster and have values obtained again from the fuzzy c-means part of the clustering step.

- Layer 3: This layer is the firing strength calculation layer. Since each fuzzy rule's antecedent part has AND connection operator, the firing strengths are calculated using the product T-norm operator. The most commonly used fuzzy AND operations are intersection and algebraic product [19]. In this case, the multiplication has been used, and the output of this layer has the following form:

$$
R_{j}=\prod_{i}^{n} A_{j i}\left(x_{i}\right)
$$

The number of nodes, at this layer, is equal to the number of clusters, as it was defined by the clustering preprocessing step.

- Layer 4: This layer is the normalization layer. Each node in this layer calculates the normalized activation strength of each rule by:

$$
\bar{R}_{i}=\frac{R_{i}}{\sum_{j=1}^{c} R_{j}}
$$

The normalized activation strength is the ratio of the activation strength of a given combination to the sum of activation strengths of all combinations. It represents the contribution of a given combination to the final result.

- Layer 5: This layer is related to the defuzzification /output part of the CFWNN. Each node at this layer combines the output of each node in $\mathrm{L}_{4}$ by algebraic sum operation after being multiplied by the output weight value $w_{i j}$ :

$$
O_{i}=\sum_{j=1}^{c} w_{i j} \bar{R}_{j}
$$

\section{A. Clustering-based Initialization}

The applied clustering algorithm at layer L2 consists of two stages [13]. In the first stage the method similar to Learning Vector Quantization (LVQ) algorithm generates crisp cpartitions of the data set. The number of clusters $\mathrm{c}$ and the cluster centers $v_{i}, i=1, \ldots, c$, obtained from this stage are used by Fuzzy c-means (FCM) algorithm in the second stage. The first stage clustering algorithm determines the number of clusters by dividing the learning data into these crisp clusters and calculates the cluster centers which are the initial values of the fuzzy cluster centers derived the second stage algorithm. Let $\boldsymbol{X}=\left[\boldsymbol{x}_{1}, \ldots, \boldsymbol{x}_{\boldsymbol{n}}\right] \in \mathrm{R}^{\mathrm{np}}$ be a learning data. The first cluster is created starting with the first data vector from $\mathrm{X}$ and the initial value of the cluster centre is taking as a value of this data vector. Then other data vectors are included into the cluster but only these ones which satisfy the following condition

$$
\left\|x_{k}-v_{i}\right\|<D
$$

where $x_{k} \in X, k=1, \ldots, n$ and $v_{i}, i=1, \ldots, c$ are cluster centers, $V=\left[v_{1}, \ldots, v_{n}\right] \in \mathrm{R}^{\mathrm{cp}}$, the constant value $\mathrm{D}$ is fixed at the beginning of the algorithm. Cluster centers $v_{i}$ are modified for each cluster (i.e., $i=1, \ldots, c$ ) according to the following equation

$$
v_{i}(t+1)=v_{i}(t)+a_{t}\left(x_{k}-v_{i}(t)\right)
$$

where $t=0,1,2, \ldots$ denotes the number of iterations, $a_{t} \in[0,1]$ is the learning rate and it is decreasing during the execution of the algorithm (depending on the number of elements in the cluster). At the end of first stage, the number of clusters $\mathrm{c}$ is defined, while the dataset is divided into the clusters. In addition, the values of cluster centers $v_{i}, i=1, \ldots, c$, which can be used as initial values for the second stage clustering algorithm, are calculated. In the second stage the fuzzy c-means algorithm has been used to optimize the values of cluster centers. 


\section{B. CFWNN Learning Phase}

The learning algorithm of CFWNN involves the use of the gradient descent (GD) method to optimize the various network parameters. For each training pair $(x, y)$, the system output $O_{i}$ is obtained in forward pass after feeding an input pattern into the network. Then the purpose of this learning phase is that, for a given $p^{\text {th }}$ training data $\operatorname{pair}\left(x_{p}, y_{p}\right)$, the parameters are adjusted so as to minimize the error function

$$
E=\frac{1}{2} \sum_{p=1}^{P}\left(D_{p}-O_{p}\right)^{2}
$$

where $P$ is the number of outputs and $D_{p}$ the desired response of the $p^{\text {th }}$ output. Variable $O_{p}$ is defined as in Eq. 6. According to the GD method, the weights in the defuzzification layer are updated by the following equation

$$
\Delta W_{i j}=-\frac{\partial E}{\partial W_{i j}}=-\frac{\partial E}{\partial O_{i}} \frac{\partial O_{i}}{\partial W_{i j}}=\left(D_{i}-O_{i}\right) \bar{R}_{j}
$$

where $i=1,2, . ., p$ and $j=1,2, . . c$ denote the number of output and normalization units respectively. The weights of the output units are updated according to the following equation

$$
W_{i j}(t+1)=W_{i j}(t)+\eta \Delta W_{i j}
$$

where $\eta$ is the learning rate. The $\mu_{i j}$ and $\sigma_{i j}$ parameters of the wavelet membership function are adjusted by the amount

$$
\begin{aligned}
& \mu_{i j}(t+1)=\mu_{i j}(t)-\eta_{\mu}\left(\frac{\partial E}{\partial \mu_{i j}}\right) . \\
& \sigma_{i j}(t+1)=\sigma_{i j}(t)-\eta_{\sigma}\left(\frac{\partial E}{\partial \sigma_{i j}}\right)
\end{aligned}
$$

$\frac{\partial E}{\partial \mu_{i j}}, \frac{\partial E}{\partial \sigma_{i j}}$ components need to be calculated using the chain rule.

$$
\begin{gathered}
\frac{\partial E}{\partial \mu_{i j}}=\frac{\partial E}{\partial \bar{R}_{j}} \frac{\partial \bar{R}_{j}}{\partial R_{j}} \frac{\partial R_{j}}{\partial A_{i j}} \frac{\partial A_{i j}}{\partial \mu_{i j}}, \\
\frac{\partial E}{\partial \sigma_{i j}}=\frac{\partial E}{\partial \bar{R}_{j}} \frac{\partial \bar{R}_{j}}{\partial R_{j}} \frac{\partial R_{j}}{\partial A_{i j}} \frac{\partial A_{i j}}{\partial \sigma_{i j}},
\end{gathered}
$$

Analytically, the partial derivatives are defined as

$$
\begin{aligned}
& \frac{\partial E}{\partial \bar{R}_{j}}=-\sum_{i=1}^{p}\left(D_{i}-O_{i}\right) w_{i j} \\
& \frac{\partial \bar{R}_{j}}{\partial R_{j}}=\frac{\sum_{i=1}^{c} R_{i}-R_{j}}{\left(\sum_{i=1}^{c} R_{i}\right)^{2}}
\end{aligned}
$$

$$
\begin{aligned}
& \frac{\partial R_{j}}{\partial A_{i j}}=\prod_{i \neq j} A_{i j} \\
& \frac{\partial A_{i j}}{\partial \mu_{i j}}=\frac{1}{1+\varepsilon} A, \\
& A=\left(\frac{\left(x_{j}-\mu_{i j}\right)}{\left(\sigma_{i j}\right)^{2}}\left(3-\frac{(x-\mu)^{2}}{\left(\sigma_{i j}\right)^{2}}\right) \exp \left(-\frac{1}{2}\left(\frac{(x-\mu)^{2}}{\left(\sigma_{i j}\right)^{2}}\right)\right)\right)
\end{aligned}
$$

and

$$
\begin{aligned}
& \frac{\partial A_{i j}}{\partial \sigma_{i j}}=\frac{1}{1+\varepsilon} B \\
& B=\left(\frac{\left(x_{j}-\mu_{i j}\right)^{2}}{\left(\sigma_{i j}\right)^{3}}\left(3-\frac{(x-\mu)^{2}}{\left(\sigma_{i j}\right)^{2}}\right) \exp \left(-\frac{1}{2}\left(\frac{(x-\mu)^{2}}{\left(\sigma_{i j}\right)^{2}}\right)\right)\right)
\end{aligned}
$$

All modeling schemes have been implemented in MATLAB (ver. R2014a, Mathworks.com).

\section{RESULTS \& DisCUSSION}

A machine learning approach based on the proposed CFWNN model has been adopted in order to create a dual model acting as an efficient classifier, in an effort to classify meat samples in three quality classes (fresh, semi-fresh, spoiled) and simultaneously as a predictor. Its structure consists of an input layer which contains six input nodes (i.e. storage temperature, sampling time, and the values of the first four principal components). The output layer consists of two nodes, corresponding to the predicted quality class (fresh, semi-fresh, spoiled) of meat samples and the related microbiological attribute, respectively. The initial enose dataset was divided into a training subset with approx. $66.5 \%$ of the data, and a testing subset with the remaining $33.5 \%$ (i.e. 70 samples). As both output parameters are not independent, in the sense that quality class is related to microbiological counts and vice versa, a model that combines both these measurements have been considered to be desirable. The real challenge in this paper is to propose a new learning-based structure which could be considered as a benchmark method towards the development of efficient intelligent methods in food quality analysis. For this reason, produced results are compared against the PLS technique, which is considered as well-recognized tool in chemometric analysis. In addition, CFWNN's prediction results are compared with those obtained by MLP networks and adaptive neuro-fuzzy inference system (ANFIS) identification models. Such schemes have become popular modeling techniques in food science and technology in recent years [14]. In the proposed CFWNN, 10 final rules have been created, using the clustering pre-processing stage. Although the classic GD method utilised as a learning scheme, the training time was completed in less than 1000 epochs, much faster from the equivalent time used to train the MLP neural network. The classification accuracy of the model was determined by the number of correctly classified 
samples in each sensory class divided by the total number of samples in the class. The performance of the model for the prediction of TVC for each meat sample was determined by the bias $\left(\mathrm{B}_{\mathrm{f}}\right)$ and accuracy $\left(\mathrm{A}_{\mathrm{f}}\right)$ factors, the mean relative percentage residual (MRPE) and the mean absolute percentage residual (MAPR), and finally by the root mean squared error (RMSE) and the standard error of prediction (SEP) [15].

TABLE II. CONFUSION MATRIX FOR CFWNN ACTING AS CLASSIFIER

\begin{tabular}{|l|c|c|c|c|c|}
\hline True class & Predicted class & \multicolumn{3}{c|}{$\begin{array}{c}\text { Row total } \\
\left(\mathrm{n}_{\mathrm{i}}\right)\end{array}$} & $\begin{array}{c}\text { Sensitivity } \\
(\%)\end{array}$ \\
\cline { 2 - 4 } & Fresh & Semi-fresh & Spoiled \\
\hline Fresh $(\mathrm{n}=16)$ & 15 & 1 & 0 & 16 & 93.75 \\
\hline Semi-fresh $(\mathrm{n}=24)$ & 0 & $22+1$ (marginal) & 1 & 24 & 95.83 \\
\hline Spoiled $(\mathrm{n}=30)$ & 0 & 0 & $29+1$ (marginal) & 30 & 100 \\
\hline Column total $\left(\mathrm{n}_{\mathrm{j}}\right)$ & 15 & 24 & 31 & 70 & \\
\hline Specificity $(\%)$ & 100 & 95.83 & 96.77 & \\
\hline Overall correct classification (accuracy): 97.14\% & \\
\hline
\end{tabular}

Results revealed that the classification accuracy of the CFWNN model was very satisfactory in the characterization of beef samples, indicating the advantage of a hybrid intelligent approach in tackling complex, nonlinear problems, such as meat spoilage. The classification accuracy is presented in the form of a confusion matrix in Table II. The model overall achieved a $97.14 \%$ correct classification, and $93.75 \%, 95.83 \%$ and $100 \%$ for fresh, semi-fresh and spoiled meat samples, respectively.

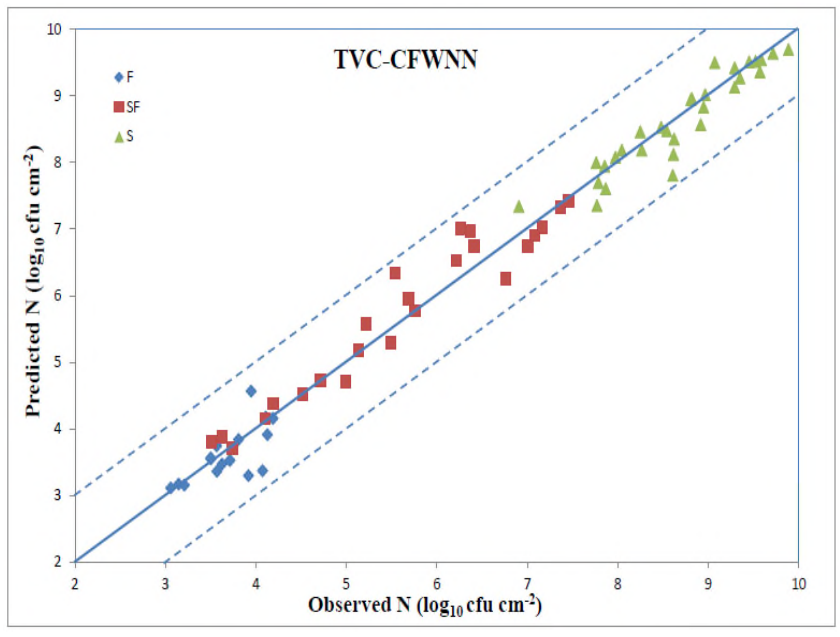

Fig. 5. CWFNN prediction model for TVC

The sensitivity (i.e. how good the network is at identifying correctly the positive samples) as well as the specificity index (i.e. how good the network is at identifying correctly the negative samples) were high, indicating satisfactory discrimination between these three classes. It is characteristic that no fresh samples were misclassified as spoiled and vice versa, indicating that the biochemical information provided by enose data could discriminate these two classes accurately.

The plot of predicted (via CFWNN) versus observed total viable counts is illustrated in Fig. 5, and shows a very good distribution around the line of equity $(\mathrm{y}=\mathrm{x})$, with all the data included within the $\pm 1 \log$ unit area. A more comprehensible picture of the CFWNN's prediction performance is however provided in Fig 6 where the \% relative error of prediction is shown against the observed microbial population.

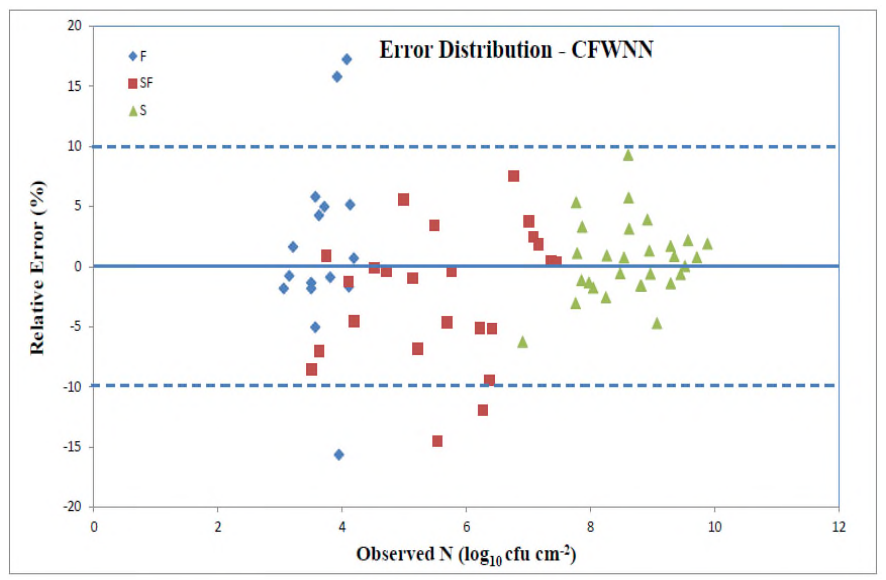

Fig. 6. CFWNN's Residual Error performance

Based on this plot, data were distributed above and below 0 , with approximately the majority of predicted microbial counts included within the $\pm 10 \%$ RE zone. Samples "6A9", "8A9", "10A9", "8A5", and "8A7" are clearly placed outside the $\pm 10 \%$ RE zone. The "6A9", "8A9", "10A9", cases correspond to AIR samples stored at the same time $\left(16^{\circ} \mathrm{C}\right)$ and collected at $18 \mathrm{~h}, 24 \mathrm{~h}$ and $30 \mathrm{~h}$ respectively. The cases "8A5" and "8A7" scorrespond to AIR samples collected at $24 \mathrm{~h}$ and stored at $10^{\circ} \mathrm{C}$ and $12^{\circ} \mathrm{C}$ respectively.

TABLE III. PERFORMANCE OF CFWNN MODEL FOR TVC

\begin{tabular}{|l|c|c|c|c|}
\hline Statistical index (CFWNN case) & Fresl & Semi-fresh & Spoiled & 0ver'all \\
\hline Mean squared error (MSE) & 0.0911 & 0.1112 & 0.0681 & 0.0881 \\
\hline Root mean squared error (RMSE) & 0.3019 & 0.3334 & 0.2610 & 0.2969 \\
\hline Mean relative percentage residual (MRPR \%) & 1.6654 & -2.2672 & 0.5252 & -0.1716 \\
\hline Mean absolute percentage residual (MAPR \%) & 5.2899 & 4.4713 & 2.3303 & 3.7409 \\
\hline Bias factor ' $B_{f}$ ) & 0.9805 & 1.0212 & 0.9943 & 1.0002 \\
\hline Accuracy factor (Af) & 1.0559 & 1.0446 & 1.0238 & 1.0382 \\
\hline Standard error of prediction (SEP \%) & 8.1776 & 5.9582 & 3.0053 & 4.5785 \\
\hline
\end{tabular}

The performance of the CFWNN model to predict TVCs in beef samples in terms of statistical indices is presented in Table III. Based on the calculated values of the bias factor $B_{f}$, it can be assumed that the proposed model underestimated TVCs in fresh and spoiled samples $\left(B_{f}<1\right)$, whereas for semi-fresh samples over-estimation of microbial population was evident $\left(B_{f}>1\right)$. The overall $B_{f}$ was almost optimal (ca. 1.0). The mean relative percentage residual index (MRPR) similarly verified the over-prediction for semi-fresh samples (MRPR < 0) and under-prediction for fresh and spoiled samples (MRPR >0). Finally, the standard error of prediction (SEP) index is a relative typical deviation of the mean prediction values and expresses the expected average error associated with future predictions. The value of 
the index was $4.57 \%$ for the overall samples, indicating good performance of the network for microbial count predictions. However in the case of fresh samples, the index gave higher values (i.e. $8.17 \%$ ).

In addition to CFWNN, in this research work, an ANFIS model has been developed to predict TVCs. The same validation technique, as well as the same training dataset has been utilized also for this case. Under these conditions, ANFIS performed satisfactory, its performance however was achieved with a high computational cost, by utilizing two membership functions for each input variables and 64 fuzzy rules. Statistical information for ANFIS model is illustrated at Table IV.

TABLE IV. PERFORMANCE OF ANFIS MODEL FOR TVC

\begin{tabular}{|c|c|c|c|c|c|c|}
\hline Statisiti & Freshl & Semi-fresh & Spoiled & $\begin{array}{l}\text { 0verall } \\
\text { ANFIS }\end{array}$ & $\begin{array}{l}\text { Overall } \\
\text { MLP }\end{array}$ & $\begin{array}{l}\text { Overall } \\
\text { PLS }\end{array}$ \\
\hline & & & 0.1821 & 0.2214 & & 1.8587 \\
\hline Rootmel & 0.4310 & 425 & 0.4267 & 0.4705 & & 1,3633 \\
\hline Mean it' & $-2,3051$ & 0791 & 0.7813 & -1.1780 & -0.4163 & 2.2667 \\
\hline Mean ab & 8.7771 & 7,9101 & 3.6923 & 6,3007 & 6.2523 & 20,1221 \\
\hline Bias factor $\left(B_{j}\right)$ & 1.0162 & 1.0245 & 0.9909 & 1.0081 & 1.0002 & 0.9946 \\
\hline Accllinacy fuctor $(A D)$ & 1.0924 & 1.0793 & 1.0381 & 1.0644 & 1.0643 & 1,2126 \\
\hline Stmidard error of prediction (SEP \%) & 11.6759 & 9,6944 & 4,9139 & 72567 & 75514 & 21.0263 \\
\hline
\end{tabular}

Finally, an MLP network and a PLS regression model were constructed for this case study. MLP was implemented with two hidden layers (with 12 and 6 nodes respectively) and two output nodes, one for the sensory class and one for the TVCs. The PLS model was constructed using the same input vector as in the cases of CFWNN and MLP, and the PLS_Toolbox software (ver. 8.0, Eigenvector.com) in association with MATLAB was used to perform the PLS analysis. The nonlinear iterative partial least squares algorithm (NIPALS) has been chosen as the appropriate learning scheme. Statistical information for both MLP and PLS models is also illustrated at Table IV.

Overall results revealed that prediction accuracy of the CFWNN model was better compared with the performances of MLP and ANFIS, in the characterization of meat samples for this reduced number of samples, indicating again the superiority of this specific MIMO hybrid intelligent modeling approach in tackling complex, nonlinear problems such as the meat spoilage. However, the produced performance from PLS scheme was expected, as it is well known that in modeling of real processes, linear PLS has some difficulties in its practical applications since most real problems are inherently nonlinear and dynamic.

\section{CONCLUSIONS}

In conclusion, this simulation study demonstrated the effectiveness of the detection approach based on electronic nose which in combination with an appropriate machine learning strategy could become an effective tool for monitoring meat spoilage during aerobic storage at various temperatures. The collected "volatile" data could be considered as biochemical "signature" containing information for the discrimination of meat samples in quality classes corresponding to different spoilage levels, whereas in the same time could be used to predict satisfactorily the microbial load directly from the sample surface. The realization of this strategy has been fulfilled with the development of a MIMO fuzzy-wavelet network which incorporates a clustering pre-processing stage. Classification performance was almost excellent, while overall prediction for TVCs has been considered as very satisfactory, although lower performance was observed especially for the fresh samples. Prediction performances of MLP and PLS schemes revealed the deficiencies of these systems which have been used extensively in the area of Food Microbiology. There is need to explore further the use of hybrid intelligent systems, and this paper has attempted for the first time to associate enose data with such systems. Further research will be focused in incorporating to the data analysis, specific microbiological data, such as Pseudomonas spp., Brochothrix thermosphacta, Lactic acid bacteria and Enterobacteriaceae.

\section{ACKNOWLEDGMENT}

The first author would like to thank Dr E.Z. Panagou from Agricultural University of Athens, Greece for providing the enose dataset, as well as the related microbiological analysis that correspond to the beef samples.

\section{REFERENCES}

[1] B. Dissing, O. Papadopoulou, C. Tassou, B. Ersbøll, J. Carstensen, E. Panagou, G-J. Nychas, "Using multispectral imaging for spoilage detection of pork meat", Food and Bioprocess Technology, vol. 6, No. 9, pp. 2268-2279, 2013

[2] Mouwen D.J.M., Hörman A., Korkeala H., Alvarez-Ordóñez A. Prieto, M., "Applying Fourier-transform infrared spectroscopy and chemometrics to the characterization and identification of lactic acid bacteria", Vibrational Spectroscopy, vol. 56, No. 2, pp. 193-201, 2011

[3] Wang, Q., Lonergan, S.M., Yu, C., "Rapid determination of pork sensory quality using Raman spectroscopy", Meat Science, vol. 91, No. 3, pp. 232-239, 2012

[4] Nychas G.-J.E., Skandamis P.N., Tassou C.C., Koutsoumanis K.P., "Meat spoilage during distribution", Meat Science, Vol. 78, pp. 7789,2008

[5] Gardner, J. W., Bartlett, P. N., "A brief history of electronic noses", Sensors and Actuators B, Vol. 18, pp. 211-220, 1994

[6] Balasubramanian, S., Panigrahi, S., Logue, C. M., Gu, H., \& Marchello, M., "Neural networks integrated metal oxide based artificial olfactory system for meat spoilage identification", Journal of Food Engineering, Vol. 91, pp. 91-98, 2009.

[7] D. Wang, X. Wang, T. Liu, Y. Liu, "Prediction of total viable counts on chilled pork using an electronic nose combined with support vector machine", Meat Science, Vol. 90, pp. 373-377, 2012

[8] X. Tian, J. Wang, S. Cui, "Analysis of pork adulteration in minced mutton using electronic nose of metal oxide sensors", Journal of Food Engineering, Vol. 119, pp. 744-749, 2013.

[9] M. Hubert, P. Ousseeuw, K. Branden, "ROBPCA: A New Approach to Robust Principal Component Analysis", Technometrics, Vol. 47, No. 1, pp. 64-79, 2005

[10] O. Papadopoulou, E.Z. Panagou, F. Mohareb, G-J. Nychas, "Sensory and microbiological quality assessment of beef fillets, using a portable electronic nose in tandem with support vector machine analysis”, Food Research International, Vol. 50, pp. 241-249, 2013.

[11] Di Natale, C., A. Macagnano, F. Davide, A. D'Amico, R. Paolesse, T. Boschi, M. Faccio, and G. Ferri, "An Electronic Nose for Food Analysis", Sensors and Actuators B, Vol. 44, pp. 521-526, 1997

[12] V.S. Kodogiannis, M. Amina, I. Petrounias, "A clustering-based fuzzy-wavelet neural network model for short-term load forecasting", Int. Journal of Neural Systems, Vol 23, No. 5, 2013.

[13] P. Jahankhani, V.S. Kodogiannis, J.N. Lygouras, I.P. Petrounias, "A Decision Support System for EEG signals based on Adaptive 
Inference Neural Networks", Journal of Computational Methods in Sciences and Engineering (JCMSE), Vol. 11, No. 4, pp. 209-225, 2011

[14] H. Yalcin, I. Ozturk, S. Karaman, O. Kisi, O. Sagdic, and A. Kayacier, "Prediction of Effect of Natural Antioxidant Compounds on Hazelnut Oil Oxidation by Adaptive Neuro-Fuzzy Inference System and Artificial Neural Network" Journal of Food Science, Vol. 76, No. 4, pp. 112-120, 2011

[15] V.S. Kodogiannis, T. Pachidis, E. Kontogianni, "An intelligent based decision support system for the detection of meat spoilage", Engineering Applications of Artificial Intelligence, Vol. 34, September 2014, pp. 23-36, Elsevier, ISSN: 0952-1976. 